\title{
Wirtschaftsethik in einem Leerraum
}

\author{
Jure Kovac
}

Die wichtigste Erkenntnis nach einem Jahrzehnt der Transformation in den ehemaligen sozialistischen Ländern Mittel- und Osteuropas ist es, daß die Einführung der Marktwirtschaft und der parlamentarischen Demokratie ein umfassender und langwieriger Prozeß ist. Es gibt längst keine Illusionen mehr darüber, daß sich jahrzehntelange Entwicklungsdefizite in einigen Bereichen über Nacht nachholen lassen.

Der Strom von Beratern aus entwickelten Wirtschaftsräumen (insbesondere aus den USA), die „Instant-Lösungen“ für den Aufbau von marktwirtschaftlichen Strukturen feilgeboten haben, wird immer spärlicher. Viele positive Erfahrungen bei der Übertragung des mangelnden Fachwissens lassen sich allerdings nicht wegleugnen. Es entstand andererseits aber auch ein großer Schaden, insbesondere dann, wenn man versucht hat, bestimmte Institutionen und Verhaltensweisen einfach in ein anderes Umfeld zu übertragen, ohne die örtlichen Gegebenheiten gekannt zu haben. Es darf nicht verwundern, daß solche Vorhaben keine echten Erfolgschancen hatten.

Diese Aussage trifft insbesondere auf die Privatisierung und freien Kapitalverkehr zu. Jene Länder, die diese Prozesse behutsam (schrittweise Öffnung für den freien Kapitalverkehr) eingeführt und die Dynamik der Veränderungen mit großer Sensibilität gesteuert haben, wurden durch die jüngsten Turbulenzen der Finanzmärkte weniger betroffen. Es gibt demnach eine bestimmte kritische Masse an Veränderungen, die eine Gesellschaft verkraften und umsetzen kann. Es ist daher unumgänglich, die Unterschiede zwischen ehemaligen sozialistischen Ländern, die sich aus ihrer Geschichte und ihrem Entwicklungsstand ergeben, zu beachten.

Die Reformländer schafften ihre einstige wirtschaftliche, politische und gesellschaftliche Ordnung ab. Das lief sehr schnell und man begann die neue demokratische Ordnung und die Marktwirtschaft mit viel Begeisterung und Ehrgeiz aufzubauen. Es stellte sich jedoch heraus, daß neue Strukturen nicht so schnell aufgebaut werden können, als die alten abgeschafft wurden. Aus diesem Grunde befinden sich ehemalige sozialistische Gesellschaften irgendwie in einem leeren Zwischenraum. Herkömmliche Einrichtungen bestehen nicht mehr, sie wären außerdem überholt, neue fehlen noch. Das schafft einen bestimmten Freiraum für unrechtmäßiges Handeln und moralisch fragwürdiges Verhalten von Einzelnen. 
Gesellschaftliche Werte und insbesondere die Wirtschaftsethik sind Bereiche, die oft in diesen Freiraum hineinfallen. Es fehlen nicht nur neue Institutionen, sondern auch die Träger, die ein gesamtheitliches ethisches Wertesystem in der Gesellschaft aufbauen würden. Es kann beobachtet werden, daß Reformländer wenig oder gar nicht bemüht sind, neue gesellschaftliche und geschäftliche Werte zu fördern. Noch schlimmer. In bestimmten Kreisen wird unethisches Handeln sogar honoriert.

Jede Gesellschaft braucht zwangsläufig Normen (auch moralische) und Institutionen, die etwaige Abweichungen von festgelegten Regeln sanktionieren können. Leider weisen die Reformländer gerade in diesem Bereich ihre größten Defizite auf bzw. sind mit dem Aufbau von Institutionen, die Verletzungen der allgemein gültigen Normen sanktionieren würden, stark im Verzug.

Es herrscht insbesondere im legislativen Bereich eine große Lücke. Der Aufbau, die Umsetzung und Überwachung der neuen Gesetzgebung kommt nur schleppend oder zu langsam voran. Es entstehen in der Folge Rechtslücken, die Freiraum für ordnungswidriges und unethisches Handeln schaffen.

Es bleibt die Hoffnung, daß diese Lücken allmählich geschlossen werden, hoffentlich mit positiven Werten, die sich aus negativen Erfahrungen der Transformationsphase herausgebildet haben. 